services, research identifying reasoning behind caregiver choices, their understanding of healthcare options and the provision of further education and health information represent worthwhile steps in tackling this issue.

\section{G107(P) THE DEVELOPMENT OF A CONSULTANT PAEDIATRICIAN PHONE-LINE FOR GENERAL PRACTITIONERS}

RC Mitchell, 0 Akindolie. Department of Ambulatory Paediatrics, Kings College Hospital NHS Foundation Trust, London, UK

\subsection{6/archdischild-2015-308599.106}

Aims Ambulatory paediatric services have changed significantly over the last year at our hospital. One of these changes has been the development of a consultant-manned phone line, whereby general practitioners (GPs) can speak directly to a consultant paediatrician between 0800-2300 on weekdays and 0800-2000 at weekends. The aim was to optimise patient care by facilitating timely communication with the most appropriate hospital based paediatrician. This enables acutely unwell children and outpatient referrals to be directed appropriately, reducing paediatric emergency department (PED) attendances and it also serves to strengthen links between primary and secondary care.

Methods Activity data for the first five months was analysed. The consultants complete a proforma for each phone call with a predefined data set. This includes the general demographics of the caller and the patient in question, as well as information about the outcomes of calls.

Results 167 calls from GPs have been taken since the phone-line began and 59 of these have been recorded onto proformas. 29\% were from GPs asking for management advice and potential referral of outpatient problems, whereas 68\% were from GPs asking about the acute management of an unwell child in the GP surgery. There were a variety of different outcomes from the calls, directing the unwell child to the most appropriate practitioner (Figure 1). Consultants taking the phone calls recorded that $33 \mathrm{ED}$ attendances and 2 admissions had been prevented following calls from GPs.
Conclusion The phone-line has been very successful, reducing PED attendances and improving pathways of outpatient referrals. Not only does this service comply with 'Facing the Future' standards, as it is consultant delivered and available at times of peak activity, but it is also in accordance with the NHS Mandate, keeping children out of hospital, in the community wherever possible. The service is in its infancy, but thus far has been instrumental in the development of ambulatory paediatric services at our hospital. It provides excellent quality of care for local children and enhances our links with local GPs.

\section{G108(P) IN PAEDIATRIC CARDIOPULMONARY ARREST ARE SUPRAGLOTTIC AIRWAY DEVICES (SAD) ADEQUATE FOR OXYGENATING AND VENTILATING?}

E Day, N Sargant. Paediatric Emergency Department, Bristol Royal Children's Hospital, Bristol, UK

\subsection{6/archdischild-2015-308599.107}

Clinical scenario A two year old has a witnessed cardiopulmonary arrest whilst in your Emergency Department. You are able to ventilate the child using a bag valve mask (BVM) and oral pharyngeal airway, but notice his stomach is becoming inflated. In cardiac arrest in adults you know that a LMA or iGel is now the advised airway to use (ALS guidelines 2011), but you wonder if this could apply to children as well.

Search parameters MEDLINE (1950-present) and EMBASE (1980 to present) [(exp ventilation) AND (exp ped* OR paed* OR child) AND (exp arrest)] LIMIT: English.

Search outcomes 327 papers, 6 of which were relevant. A further two papers were found from the reference of one of the original search articles.

Comments No paper looks specifically at using a SAD in a paediatric arrest, all the cases have been in mannequins or in anaesthetised children, simulating arrest. Hypoxia, which is often the cause, is a very important factor to overcome in paediatric arrest. By using a SAD one can undertake uninterrupted chest compressions and ventilation. The comparison with BVM and intubation in the prehospital setting shows that neurological

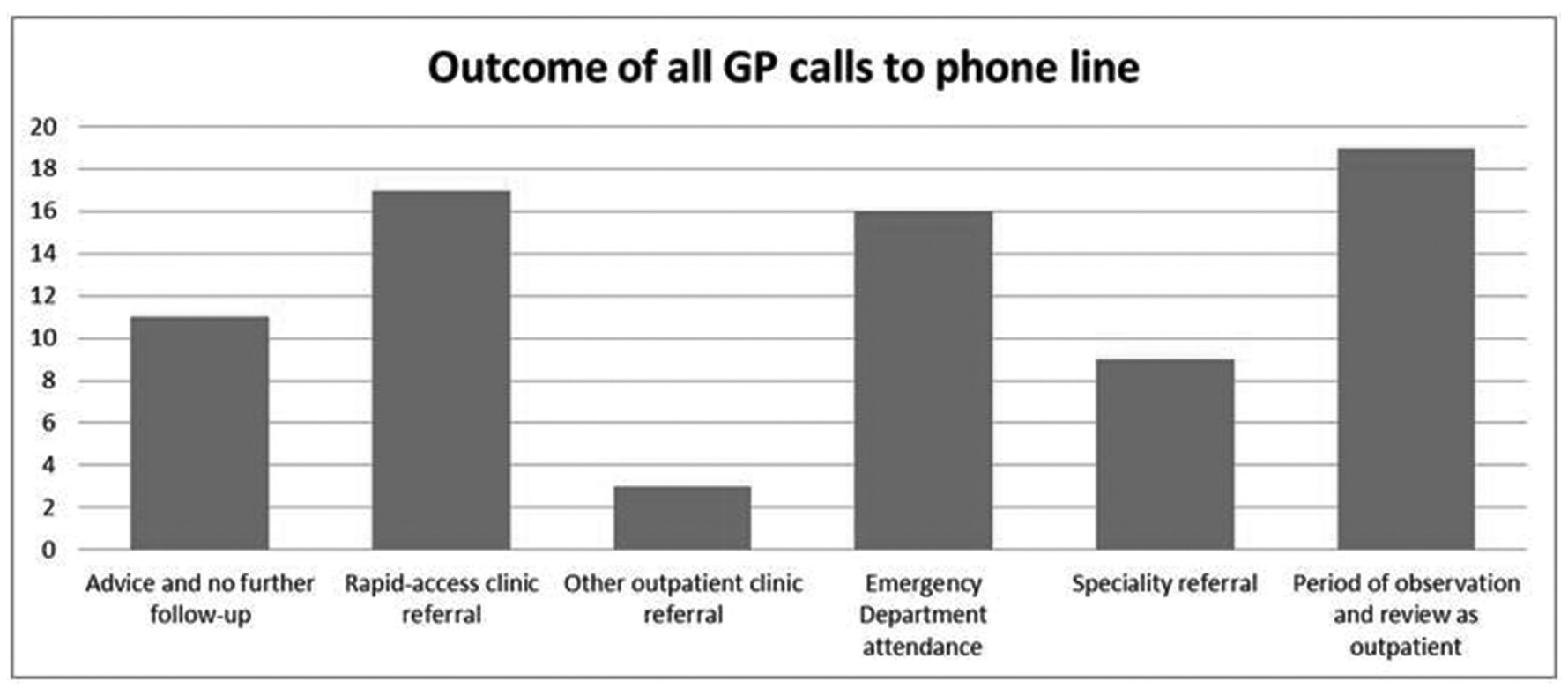

Abstract G107(P) Figure 1 The outcomes of all phone calls from GPs to the phone-line 
outcome/survival is not significantly different between the two groups. Using a SAD may not be worth doing as it takes time to insert, meaning there is no ventilation in that time. However, in children with difficult airways who intubation poses a problem, it is worth bearing in mind the use of a SAD. Over time the effectiveness of BVM decreases, hence a more definitive airway should always be planned.

Clinical bottom line A bag valve mask with oropharyngeal airway should be used initially to oxygenate and ventilate a child in cardiopulmonary arrest. A supraglottic airway should be considered in children with a difficult airway or if there is going to be delay in establishing a definitive airway (endotracheal intubation).

SAD - supraglottic airway device. LMA - laryngeal mask airway. OPA - oropharyngeal airway. BVM - bag-valve-mask. ALS - advanced life support.

\section{British Association of Perinatal Medicine}

\section{G109 RESTRICTING VISITORS TO THE NICU SIGNIFICANTLY REDUCES NOSOCOMIAL VIRAL RESPIRATORY TRACT INFECTIONS IN BABIES}

${ }^{1}$ A Lakshmanan, ${ }^{2} \mathrm{~L}$ Szatkowski, ${ }^{1} \mathrm{~S}$ Zinna, ${ }^{2} \mathrm{M}$ Clarkson, ${ }^{2} \mathrm{R}$ McClaughry, ${ }^{1} \mathrm{~S}$ Soo, ${ }^{2} \mathrm{D}$ Sharkey. ${ }^{1}$ Department of Neonatal Medicine, Nottingham University Hospital NHS Trust, Nottingham, UK; ${ }^{2}$ Division of Academic Child Health, University of Nottingham, Nottingham, UK

\subsection{6/archdischild-2015-308599.108}

Introduction Recent data suggest $8-52 \%$ of babies on the NICU have evidence of a viral respiratory tract infection (VRTI) (Ronchi 2014, Bennett 2012). These studies, and our own data, indicate babies with VRTIs spend twice as long in hospital and have significantly worse respiratory outcomes such as chronic lung disease and the need for home oxygen. There is little evidence exploring ways of reducing these infections in the NICU. Our recent survey demonstrates significant variation in UK NICU visiting practices and isolation policies for babies with RVTIs.

Aim To establish the impact of visitor restriction on the incidence of NICU VRTIs.

Methods We performed a retrospective study of all admissions between 2007 and 2013 at two large UK tertiary NICUs $(\sim 13,300$ bed days/year). Normal visiting policy included parents, family and friends. During the periods November to April of 2009, 2010 and 2011, in response to the H1N1 pandemic, we restricted visiting to parents/carers only. No other variations in practice occurred. We identified all babies positive for VRTIs. We used a Poisson generalised additive model (GAM), factoring in workload intensity and incidence of community VRTIs, to calculate the impact of these 3 winter restriction periods compared with normal visiting.

Results There were 100 PCR proven VRTIs in 93 babies during this period $(\sim 16 / \mathrm{yr})$. Rhinovirus $(\mathrm{n}=71)$, RSV $(\mathrm{n}=8)$ and H1N1 $(n=5)$ were the most common. The median gestation of infected babies was 29 weeks (IQR 26-34 Weeks) and 46\% required an escalation of respiratory support. Two of five H1N1 positive babies died. The results from the GAM suggest there was a $39 \%$ reduction $(P<0.05)$ in VRTIs during restricted visiting periods compared to normal visiting (Incident Rate Ratio 0.61, 95\% CI 0.38-0.99). Extrapolating this to the UK, based on the NHS NICU tariff, the extra bed days associated with VRTIs cost between $£ 7 \mathrm{M}$ and $£ 25 \mathrm{M}$ /year.

Conclusion This is the first study demonstrating a significant reduction in NICU VRTIs through restricting visiting practices. VRTIs are associated with significant neonatal respiratory morbidity and have short and long-term resource implications. We need to explore better ways of minimising the impact of VRTIs in this vulnerable population.

\section{G110 THE VALIDITY OF STANDARDISED TWO-YEAR NEURODEVELOPMENTAL STATUS ASSESSED DURING ROUTINE NHS FOLLOW-UP OF CHILDREN BORN $<30$ WEEKS' GESTATION}

${ }^{1} \mathrm{HS}$ Wong, ${ }^{2} \mathrm{FM}$ Cowan, ${ }^{2} \mathrm{~N}$ Modi. Medicines for Neonates Investigator Group; ${ }^{1}$ Neonatal Intensive Care Unit, Cambridge University Hospitals NHS Foundation Trust, Cambridge, UK; ${ }^{2}$ Section of Neonatal Medicine, Department of Medicine, Imperial College London, London, UK

\subsection{6/archdischild-2015-308599.109}

Aim To determine the validity of standardised, routinely recorded NHS assessments in identifying neurodevelopmental impairments at age two years in children born <30 weeks' gestation.

Methods Children born $<30$ weeks' gestation, attending a routine NHS follow-up assessment at age 20-28 months from term, in 13 participating hospitals were invited to join the study. Data on neurodevelopmental outcome were recorded in a standardised format developed by the Thames Regional Perinatal Outcomes Group and entered into the electronic clinical record on the Badgernet platform. Data were extracted quarterly and held in the National Neonatal Research Database at the Neonatal Data Analysis Unit. Based on a predefined algorithm, each participant was classified as having 'no', 'mild-moderate' or 'severe' neurodevelopmental impairment in cognitive, language and motor domains. Participants also received a formal neurodevelopmental assessment conducted to research standards by a single assessor using the 3rd edition of the Bayley Scales (Bayley-III). The sensitivity and specificity of NHS assessments in identifying children with any impairment (Bayley-III scores $<85$ ) or severe impairment (Bayley-III scores $<70$ ) in each of the 3 domains were calculated.

Results One hundred and ninety children born at a median (IQR) gestational age of 27 (26-29) weeks participated. The validity of routine assessment in identifying children with no impairment was high across all domains (specificities 83.9$100.0 \%$ for no impairment; $96.6-100.0 \%$ for no severe impairment). The sensitivity of routine assessment in identifying gross motor impairment was also high, particularly for severe impairment. However, the identification of cognitive impairments (sensitivities (95\% CI) were $69.7 \% \quad(55.1-84.3 \%)$ for any impairment; $28.6 \%(5.0-52.2 \%)$ for severe impairment) and language impairment (53.2\% (42.0-64.5\%) for any impairment; $42.9 \%(14.2-71.5 \%)$ for severe impairment) were poor.

Conclusions Routine NHS assessments identify children with severe motor impairment with good specificity but lack adequate sensitivity in cognitive and language domains, areas where early intervention improves educational and social outcomes. About 7000 children are born $\leq 30$ weeks' gestation each year in the UK. This study emphasised that follow-up assessments should performed by appropriately trained personnel using sensitive and 